\title{
Acute posterior multifocal placoid pigment epitheliopathy
}

\section{Pigment epitheliopathy or choriocapillaritis}

\author{
A. F. DEUTMAN, J. A. OOSTERHUIS, T. N. BOEN-TAN, AND \\ A. L. AAN DE KERK \\ From the Eye Hospital, Rotterdam Medical Faculty, and the Eye Department, Leiden University \\ Hospital, The Netherlands
}

Gass (1968) presented the clinical and fluorescein angiographic findings in three young female patients showing rapid loss of central vision secondary to multifocal, yellow-white, placoid lesions at the level of the pigment epithelium and choroid; rapid resolution of these lesions with permanent alterations in the pigment epithelium and minimal damage to the adjacent choroid and retina; and significant visual improvement after apparent ophthalmoscopic resolution of the acute lesion. The lesions were located at the posterior pole, and despite extensive general physical examination and blood studies no aetiology could be found. Gass wondered about the significance of a positive tuberculin skin test in two patients and of a family history of tuberculosis in the third patient.

Maumenee ( 1970 ) reported having seen six patients with this condition and described it as one of the clinical entities discernible among the intraocular inflammations termed "uveitis". Van Buskirk, Lessell, and Friedman (I97I) described one patient with this condition who also had erythema nodosum.

Little is known about this affection, and since we have observed three young females with almost identical lesions we thought it worthwhile to present their cases. The significance of erythema nodosum with histologically demonstrated vasculitis in one of our patients is discussed with regard to the primary site of this so-called acute posterior multifocal placoid pigment epitheliopathy.

\section{Case reports}

Case 1, a 20-year-old white female, was admitted to the Rotterdam Eye Hospital on June 7, 1968, with the complaint of seeing flecks in both eyes for a week.

Examination

The visual acuity in both eyes was $\mathrm{O}_{1} \mathrm{I}$ and there was marked metamorphopsia. Both anterior chambers were clear, and the vitreous body showed a moderate number of cells in both eyes. The fundi showed multiple, generally round, whitish placoid patches with fuzzy margins scattered 
throughout the posterior pole (Fig. $1 a, b$ ). The lesions were slightly smaller than disc-size and did not extend beyond the equator.

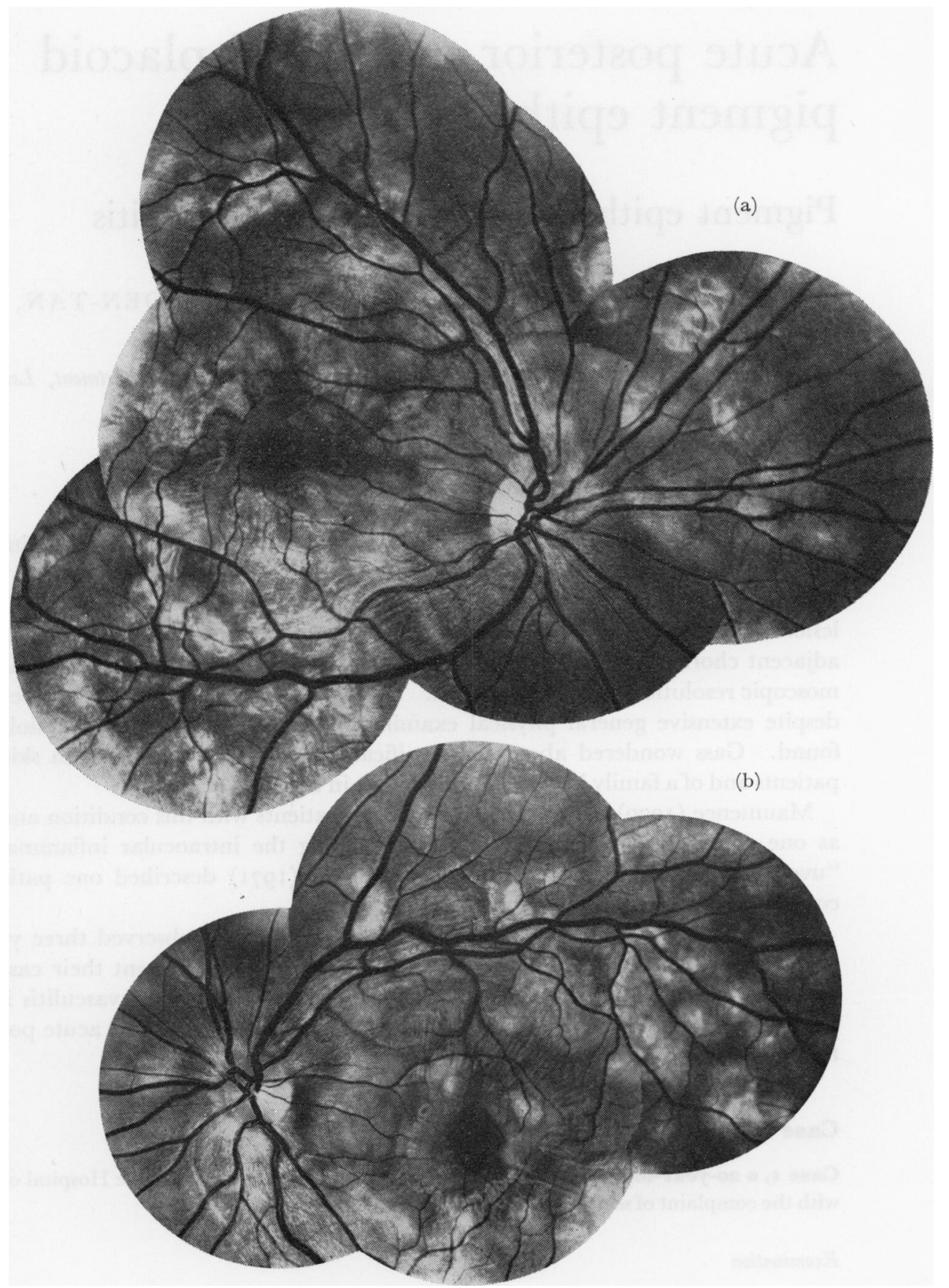

FIG. I $a, b$ Acute multifocal lesions in both eyes of Case $\mathrm{I}$ on June 7, 1968. The left eye shows more acute lesions, and early resolution of lesions is seen in the right eye 
In the right eye they had already begun to resolve. Those adjacent to the fovea showed the greatest degree of resolution, manifested by loss of the yellow-white opacification, fine pigment mottling, and depigmentation of the pigment epithelium. Fluorescein angiography showed absence of the normal choroidal fluorescence at the site of the lesions in the arterial and arterio-venous phases. There was a geographical pattern of fluorescence and non-fluorescence in the arterio-venous phase and staining at the site of the lesions in the late phases (Fig. $2 a, b, c, d)$.

(a)

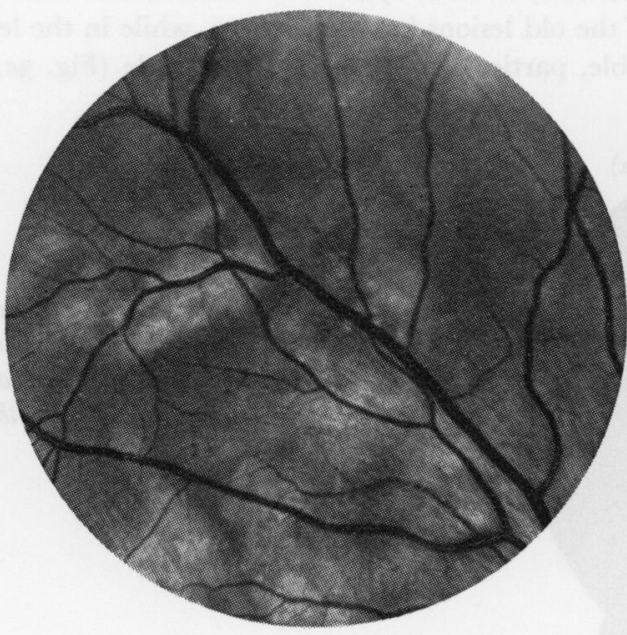

(c)

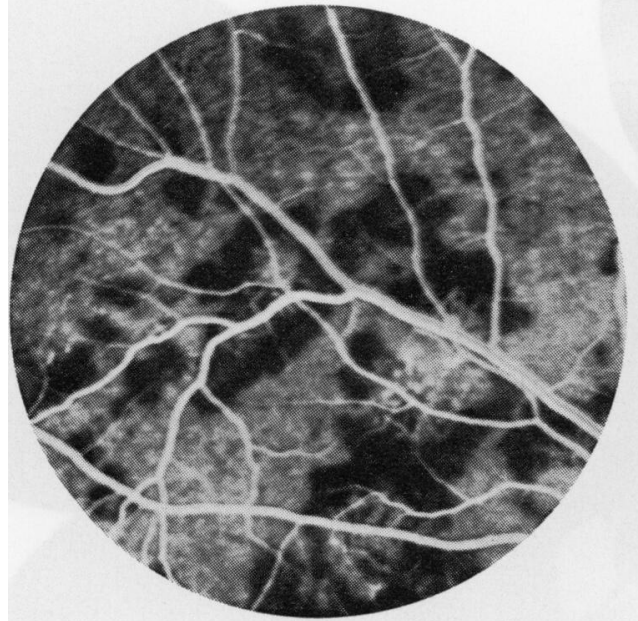

(b)

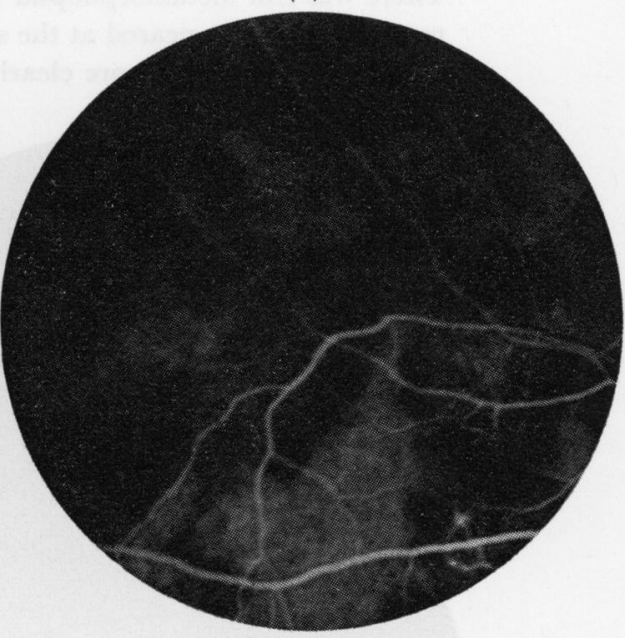

(d)

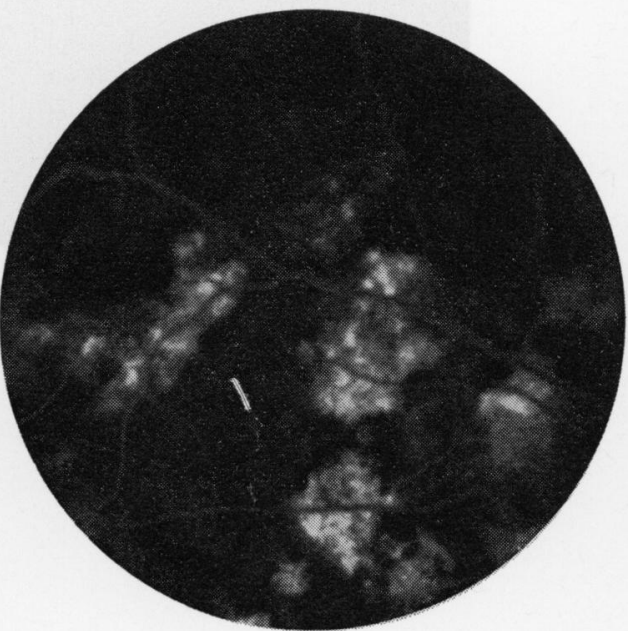

FIG. 2a-d Fluorescein angiography of right eye of Case I on June 7, I968

In the early arterial phase the choroidal fluorescence is not visible at the site of the acute lesions (Fig. 2b)

In the arterio-venous phase more choroidal fluorescence is visible, but at the site of some of the more oedematous lesions the normal choroidal background fluorescence is still obscured (Fig. 2c)

In the late venous phase there is hyperfluorescence due to staining of the lesions (Fig. 2d)

\section{Therapy}

Cortisone and vibramycin were given.

\section{Course}

On June 12, the fundi showed distinct resolution at the site of most of the lesions. The visual acuity was 0.7 in the right eye and 0.3 in the left. 
The visual fields showed several sharply delineated scotomata, corresponding to the lesions at the posterior poles. The peripheral limitations of the fields were normal.

On June 20, the visual acuity was 0.9 in the right eye and 0.4 in the left. The vitreous was clear and the lesions were resolved and sharply defined. The patient was discharged from hospital.

\section{Progress}

Two years later, on August 17, 1970, the visual acuity was 0.9 in the right eye and 0.4 in the left. There was still metamorphopsia (Amsler test) in both eyes. No active lesions were present. Pigmentations had appeared at the site of the old lesions in the right eye, while in the left eye areas of depigmentation were more clearly visible, particularly at the posterior pole (Fig. $3 a, b$ ).

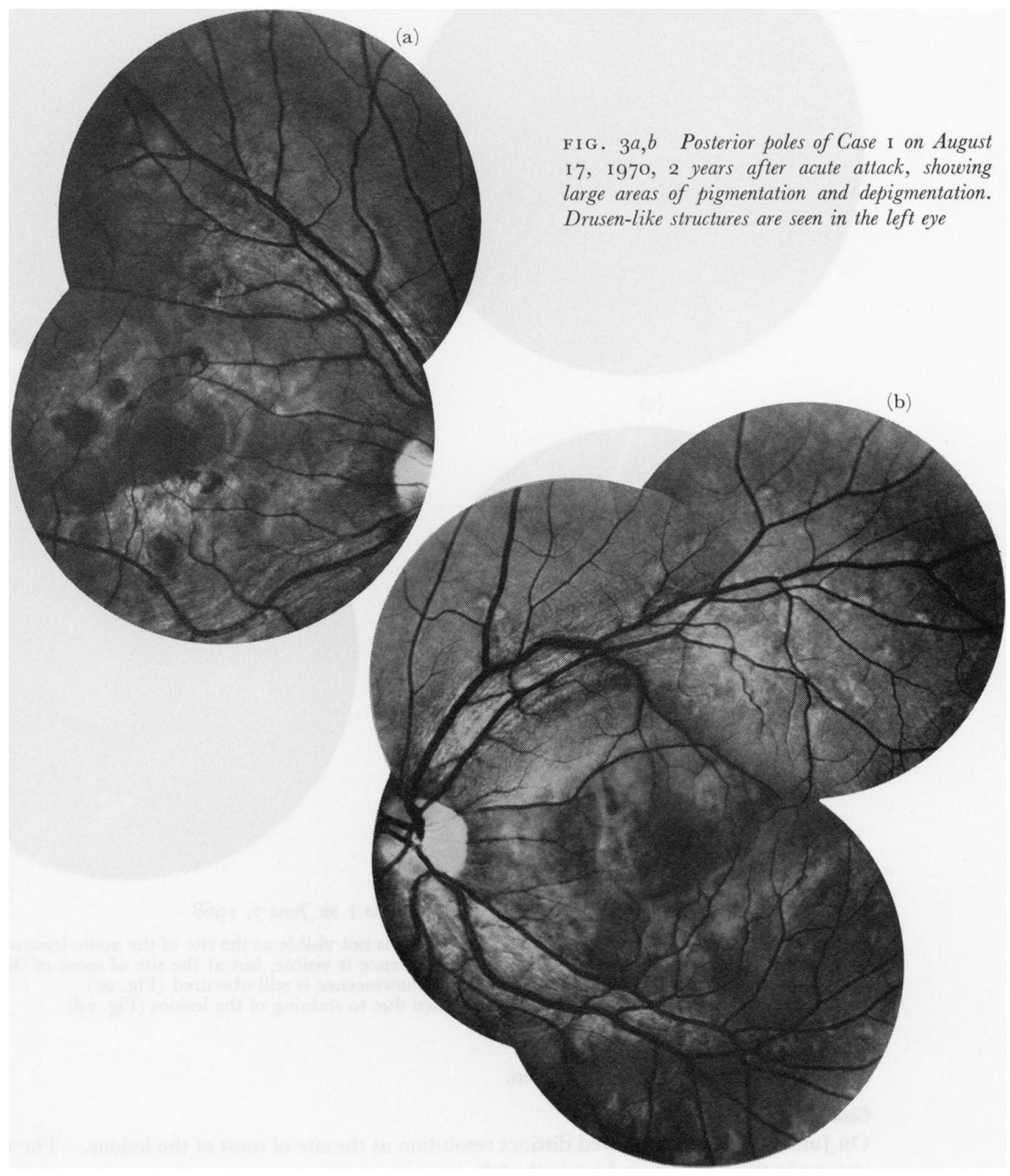


Fluorescein angiography showed extensive areas of atrophic pigment epithelium, much more than could be predicted from ophthalmoscopic examination alone (Fig. 4). There was no longer any evidence of oedema.

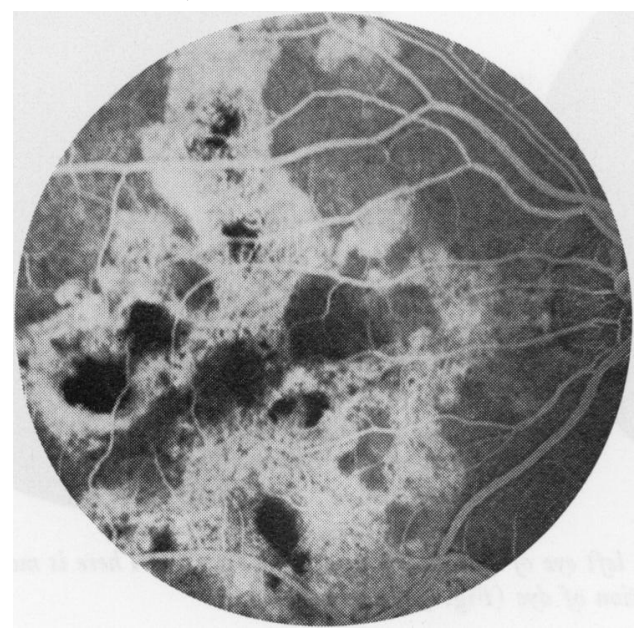

FIG. 4 Fluorescence photograph in early venous phase of right eye of Case I on August I 7, 1970, showing enormous defects in retinal pigment epithelium. The fluorescence at the site of the dense pigmentations is obscured. There is possibly some mild atrophy of the choriocapillaris

An extensive general physical examination showed no distinct abnormalities. The capillary resistance was normal. Radiology of the chest showed no abnormality. The tuberculin skin test was negative, although the girl's father had suffered from tuberculosis. The electroretinogram was normal. The only pathological finding was an abnormally high anti-streptolysin titre (700 $\mathrm{U}$, compared to the normal upper limit of $300 \mathrm{U}$ ).

Case 2, a 25-year-old white female, visited her ophthalmologist on April 8, 1970, complaining of blurred vision.

\section{Examination}

The visual acuity in both eyes was I 0 . However, metamorphopsia (Amsler test) was present, and around the foveal areas there were some oedematous chorio-retinal lesions. The next day the visual acuity had dropped to $0 \cdot 3$ in both eyes.

The patient was admitted to the eye department of the Leiden University Hospital. Both posterior poles showed fuzzy, greyish-white areas. Fluorescein angiography was performed on April ro. At the site of the lesions no normal choroidal fluorescence was visible in the arterial and arterio-venous phases, but after Io minutes the lesions showed marked staining (Fig. 5a,b).

On April Io, the visual acuity in both eyes was $\mathrm{I} \cdot 0$.

\section{Therapy}

Prednisone and isoniazid were given.

Course

The next day the visual acuity of the right eye had decreased to counting fingers at $\mathrm{I} \mathrm{m}$., and that of the left eye was $0 \cdot r$. The fundi showed roundish areas of oedema. The vitreous and anterior chambers were clear. Several days later irregular pigmentation occurred at the posterior poles and resolution of the lesions began centrally, spreading peripherally. On April I6, vision had improved to 0.2 in both eyes, and on May 5 was 0.5 .

On April I4 and 21, the visual fields showed sharply defined, round, paracentral and central scotomata corresponding to the lesions seen with the ophthalmoscope. Fluorescein angiography was repeated on May 14. There was hyperfluorescence due to defects in the retinal pigment epithelium, but no evidence of chorio-retinal staining. On May 26, the visual acuity was 0.5 in the right eye and 0.9 in the left. 


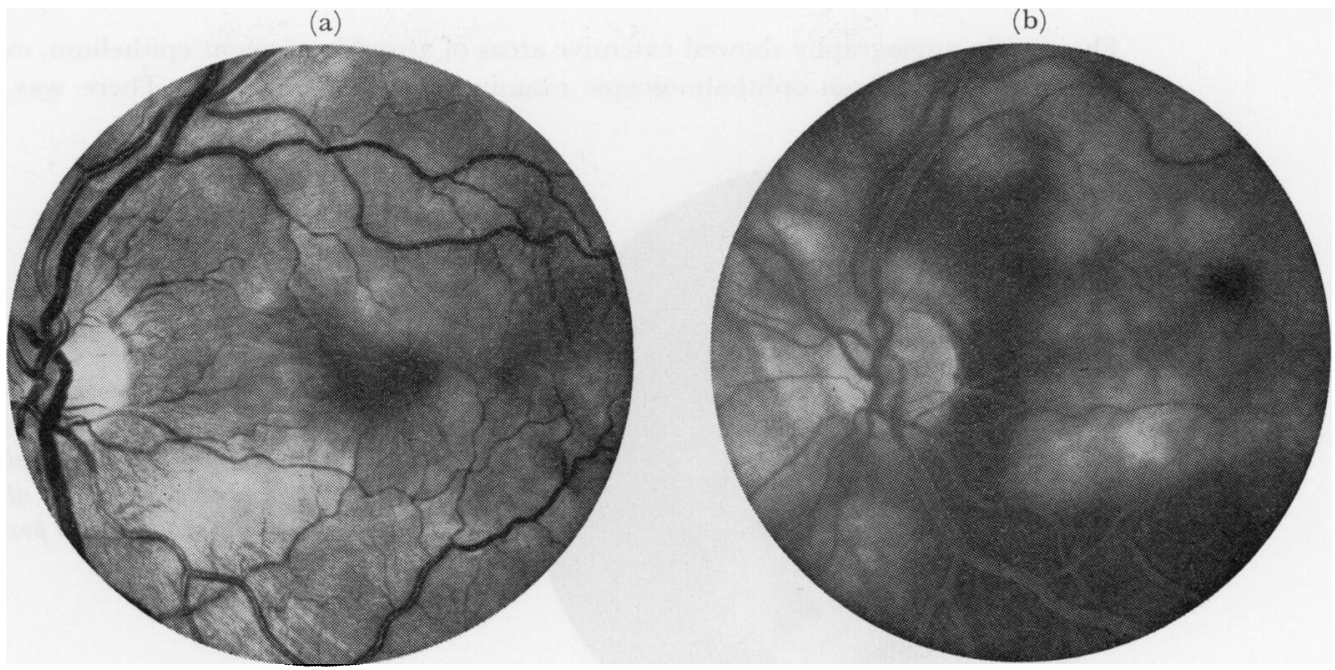

FIG. 5a,b Fluorescence photographs of the left eye of Case 2 on April 10, 1970. There is marked staining of the oedematous lesions 15 minutes after injection of dye (Fig. $5^{b}$ )

General physical examination revealed an initially raised erythrocyte sedimentation rate, which fell during the next week : 42/6 I mm. at I and 2 hrs on April Io;6/6 I on April 21. Serological examination was negative.

The antistreptolysin titre was $100 \mathrm{U}$ and the tuberculin skin test (purified protein derivative) was negative. Radiography of the chest showed no abnormality.

The only medication used by the patient was an oral contraceptive (Lyndiol 2,5). Because painful reddish infiltrates were found in both axillae and on the legs, a diagnosis of erythema nodosum was made. After skin biopsy, histological examination showed vasculitis with deep infiltrates of lymphocytes and histiocytes. The vessel endothelium was swollen. Immunofluorescence examination gave no specific results.

There was no indication of lupus erythematosus. Blood tests, blood glucose, protein spectrum, serum minerals, Meinicke, Waaler-Rose, and latex tests, toxoplasmosis complement-fixation reaction, Paul-Bunnell reaction, and urine culture (Loewenstein) were all normal. The Sabin-Feldmann test was positive $(1: 128)$ and the cytomegaly virus reaction was positive $(1: 32)$; both results being within the normal range.

Case 3, a 25-year-old white female, attended the Rotterdam Eye Hospital on June 25, 1970, complaining of blurred vision in the left eye of I week's duration. The visual acuity at that time was 0.8 in the right eye and counting fingers at $\mathrm{I} \mathrm{m}$. in the left.

At the age of 8 years she had undergone a strabismus operation on the right eye. Her vision had been normal throughout her life and had been $1 \cdot 0$ in both eyes on November 28,1969 .

\section{Examination}

On June 25, 1970, the intraocular pressure was normal in each eye. The anterior chamber of the right eye showed some cells, while the vitreous was clear. The anterior chamber and vitreous of the left eye both showed some cells. At both posterior poles there were fresh choroiditis-like patches, more in the left eye than the right. There was evidence of an old choroiditis scar in the left eye.

\section{Therapy}

Oral cortisone and vibramycin were given. 


\section{Course}

The next day the lesions were scattered all around both posterior poles (Fig. 6a,b). The right eye showed lesions not unlike large soft fresh photocoagulates; the left eye had fresh lesions around the posterior pole with resolution of the lesions in the central part of the retina, forming a scalloped pattern. Fluorescein angiography at this time showed absence of the normal choroidal fluorescence at the site of the lesions, but hyperfluorescence in the late phases.

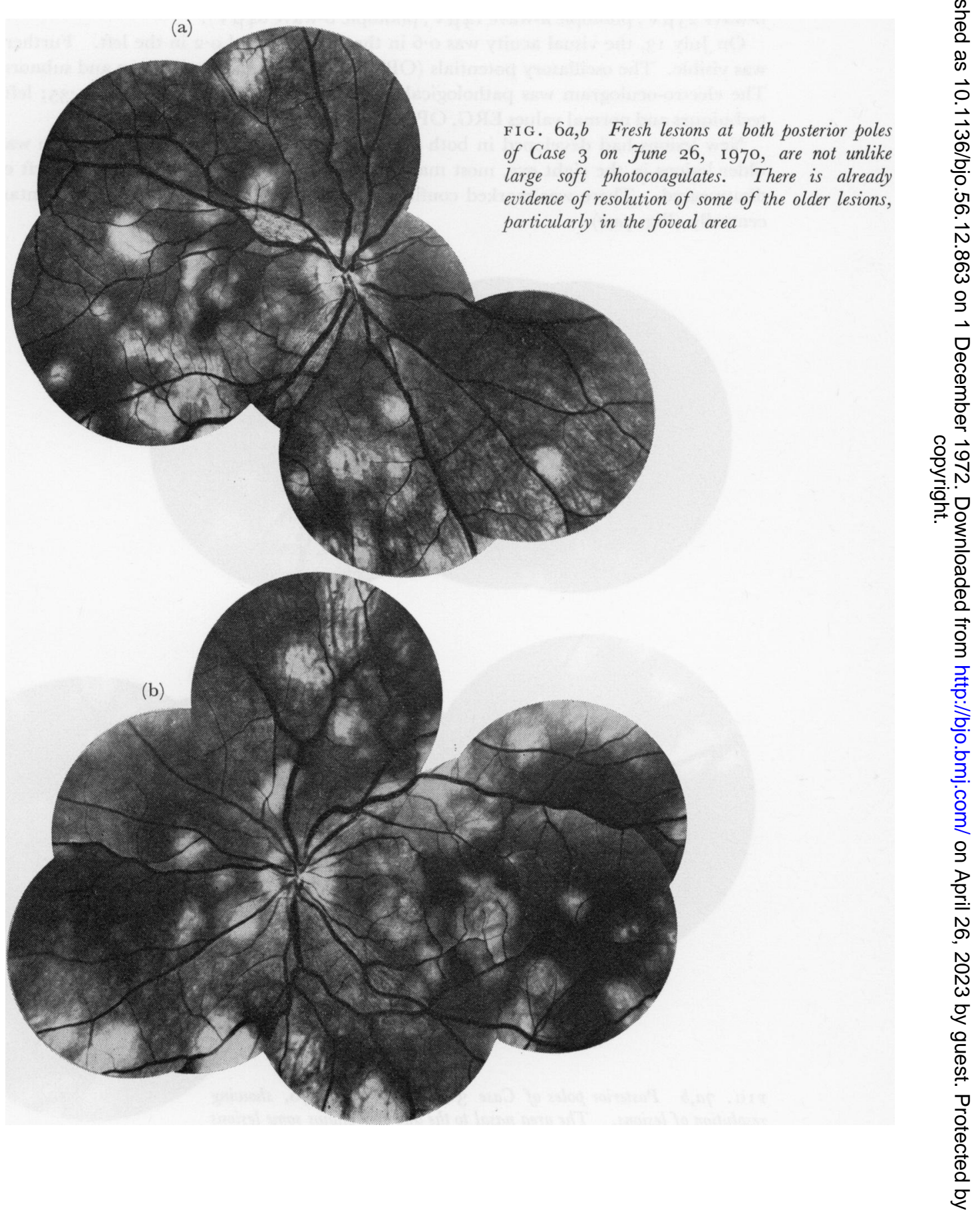


On July 2, the visual acuity had changed to 0.5 in the right eye and counting fingers at $5 \mathrm{~m}$. in the left. The visual fields showed multiple round scotomata centrally.

On July 7 , the fundus lesions showed some resolution. There was already some degree of pigmentation and depigmentation at the site of the lesions. Some new lesions had appeared around the posterior pole. The visual acuity was now 0.6 in the right eye and counting fingers at $1 \mathrm{~m}$. in the left. On July 9, the electroretinogram showed low-normal values for the right eye (scotopic b-wave I $80 \mu \mathrm{V}$; photopic a-wave $38 \mu \mathrm{V}$; photopic b-wave $\mathrm{I09} \mu \mathrm{V}$ ) and subnormal values for the left (scotopic b-wave $23 \mu \mathrm{V}$; photopic a-wave $\mathrm{I} 4 \mu \mathrm{V}$; photopic b-wave $64 \mu \mathrm{V}$ ).

On July ${ }_{13}$, the visual acuity was 0.6 in the right eye and 0.2 in the left. Further pigmentation was visible. The oscillatory potentials (OP) were normal in the right eye and subnormal in the left. The electro-oculogram was pathological in both eyes: L/D-ratio right eye $\mathrm{I} \cdot 35$; left eye $\mathrm{I} \cdot \mathrm{Io}$ (for techniques and normal values ERG, OP, and EOG: see Deutman, 1971).

New lesions had developed in both eyes since the initial examination. There was resolution of older lesions in the right eye, most marked centrally. The fresh lesions in the left eye had totally disappeared. There was marked confluence of the patches and extensive pigmentary disturbance centrally (Fig. $7 a, b)$.
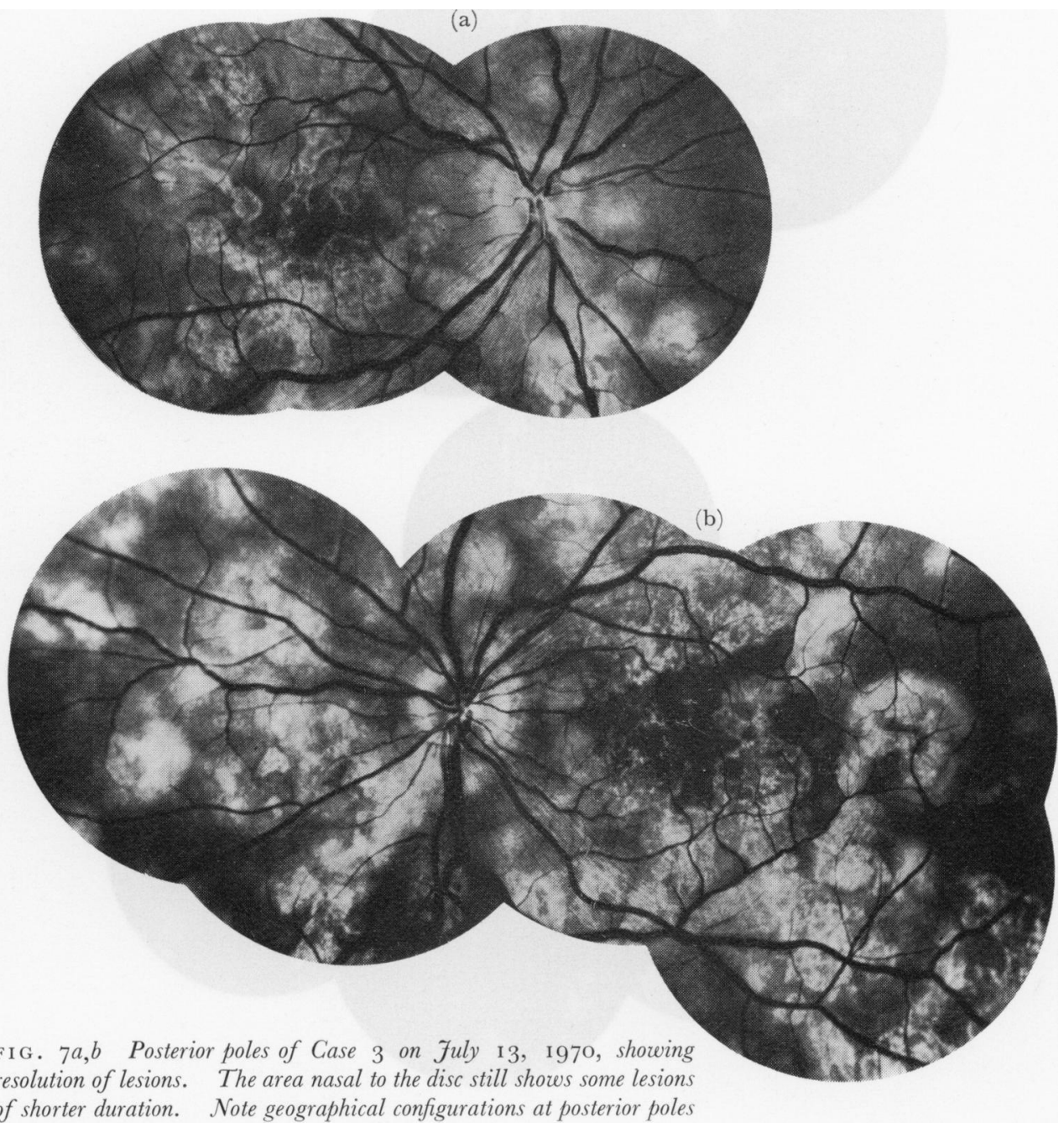

resolution of lesions. The area nasal to the disc still shows some lesions

of shorter duration. Note geographical configurations at posterior poles 
(a)

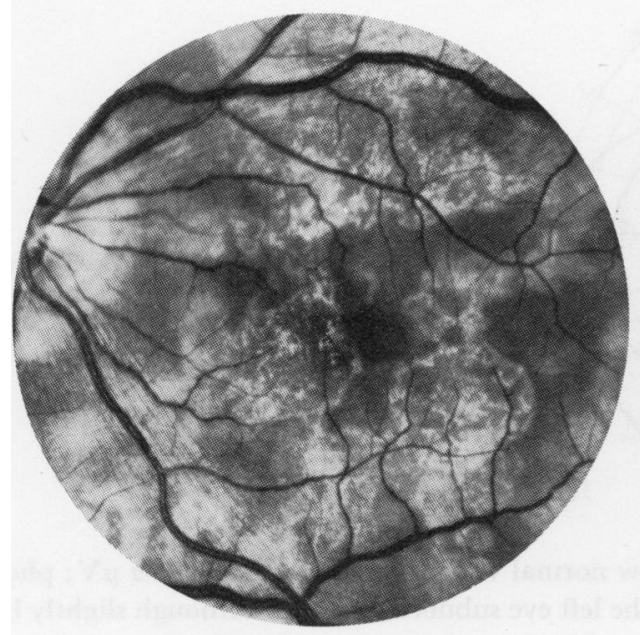

(c)

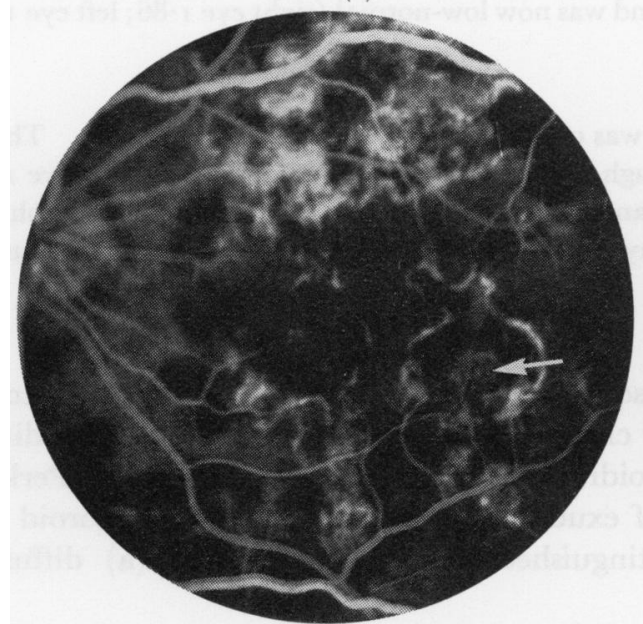

(b)

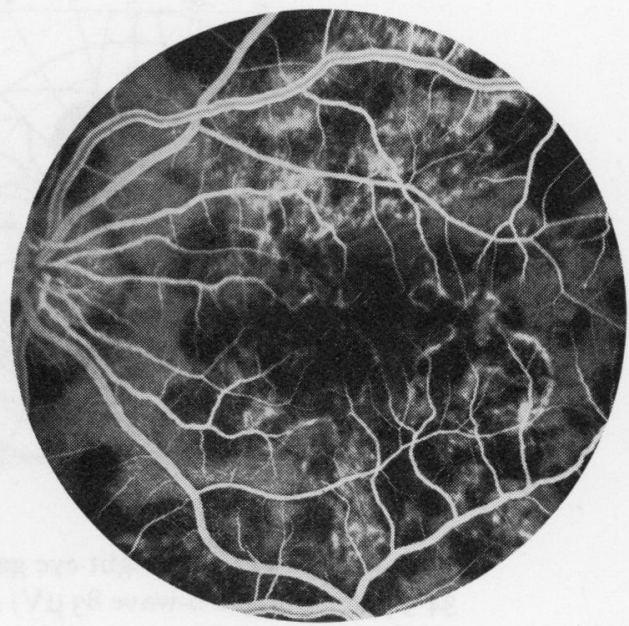

(d)

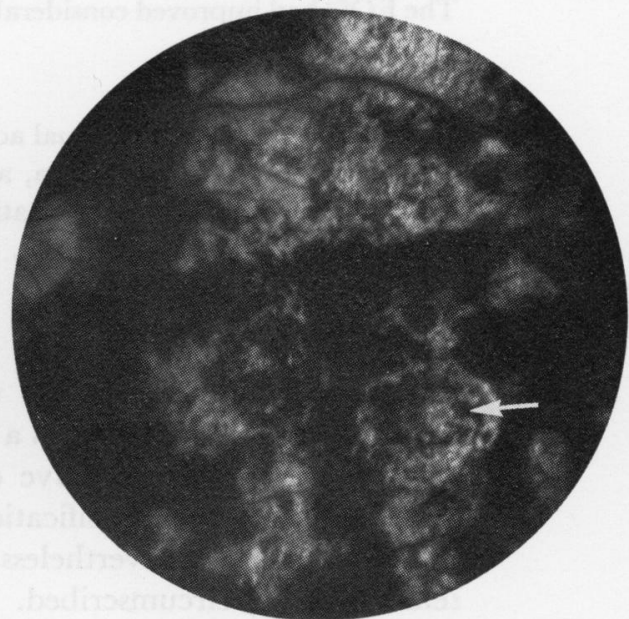

FI G. 8a,b,c,d Fluorescence angiography of left eye of Case 3, showing defective pigment epithelium and pigment scarring. There is no oedema centrally. The area temporal to the fovea shows atrophy of the choriocapillaris indicating that the pigment epithelium is not the only structure affected

Fluorescein angiography now showed defects in the pigment epithelium and patches of choroidal atrophy. There was no longer any evidence of staining (Fig. 8a,b,c,d). The visual fields, examined on July ${ }_{15}$, showed multiple round scotomata in both eyes (Fig. 9). On July 16, 1970, the patient was discharged from hospital.

Extensive general physical examination had shown no distinct abnormalities. Chest $X$-rays and the tuberculin skin test (PPD) were negative, as were all the serological reactions. At the time of admission the erythrocyte sedimentation rate was $20 \mathrm{~mm}$./ Ist hour. Neurological examination was also normal, except for a mildly and diffusely disturbed EEG; when this was repeated on August $3^{\mathrm{I}}$, the results were the same as in July during the acute stage.

The only medication used by the patient was an oral contraceptive (Gynovlar) which she had taken regularly over the past 3 years.

\section{Progress}

The ERG was repeated on September I, 1970, and showed almost the same results as in the acute 


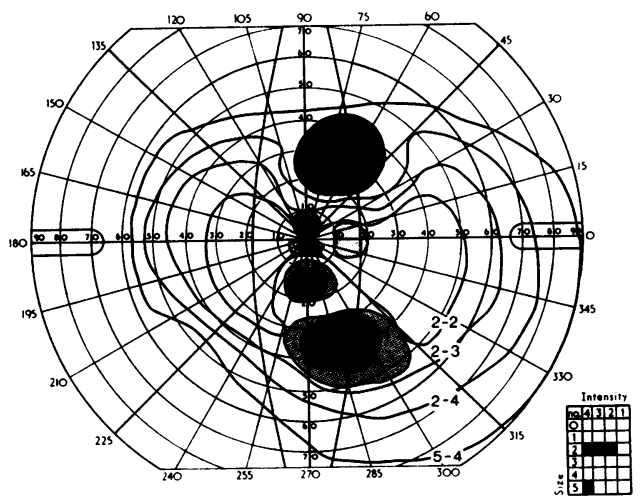

FIG. 9 Visual field of Case 3, a month after onset of symptoms, showimg multiple roundish central scotomata

stage on July 9. The right eye gave low normal values (scotopic b-wave $182 \mu \mathrm{V}$; photopic a-wave $34.9 \mu \mathrm{V}$; photopic b-wave $83 \mu \mathrm{V}$ ) and the left eye subnormal values, although slightly higher than at the first examination (scotopic b-wave $73 \mu \mathrm{V}$; photopic a-wave $22 \cdot 9 \mu \mathrm{V}$; photopic b-wave $5 \mathrm{I} \cdot 8 \mu \mathrm{V}$ ). The EOG had improved considerably and was now low-normal (right eye I $\cdot 86$; left eye $\mathrm{I} \cdot 77$ ).

Result

On January I I, I97 I, the visual acuity was 0.8 in the right eye and 0.4 in the left. The patient still complained of metamorphopsia, although the fundi showed a resolved and inactive aspect with a geographical pattern of pigmentations and de-pigmentations. Some choroidal atrophy was visible. The anterior chambers were clear. Tiny white opalescent spots were seen in the vitreous.

\section{Comment}

The question arises whether the so-called acute posterior multifocal placoid pigment epitheliopathy (APMPPE) is a new clinical entity. In our opinion, this condition belongs to the category of exudative choroiditis described by Duke-Elder and Perkins (I966); he stated that "the classification of exudative inflammations of the choroid is most unsatisfactory", but nevertheless distinguished the following types: (a) diffuse; (b) disseminated; (c) circumscribed.

(a) The description of diffuse choroiditis resembles most clearly the condition described here, but a vitreous haze is reported and white scarred areas are left in which the larger choroidal vessels may form a network. These features are not generally found in APMPPE.

(b) Disseminated choroiditis, according to Duke-Elder, always includes vitreous opacities, the entire fundus being affected. Atrophic discs, constricted vessels, and complicated cataract may eventually develop. In APMPPE the vitreous remains clear apart from the presence of some cells. Only the posterior pole is affected; the discs, vessels, and lens remain unchanged.

(c) As Gass (1968) stated, APMPPE is unlike typical focal choroiditis (circumscribed exudative choroiditis):

(I) The overlying pigment epithelium is diffusely involved, and no part of the lesions appears to lie deep to or extend laterally beyond the area of pigment epithelium.

(2) The lesions both large and small are flat or placoid.

(3) The absence of overlying serous detachment of the retina is remarkable in view of the intensity of the lesion and the reduction in visual function. 
(4) The very rapid resolution of the lesions and the absence or near absence of apparent damage to the underlying choroid despite advanced alterations in the pigment epithelium are atypical for the usual focal choroiditis such as is seen in presumed histoplasmosis or toxoplasmosis.

Reviewing these different types of acute inflammations, we can conclude that APMPPE resembles "diffuse choroiditis" in most though not in all respects. Maumenee (1970), reflecting a similar opinion, classified APMPPE as a distinct clinical entity under the heading of "uveitis".

The ophthalmoscopical and fluorescein pattern in the acute stage is identical to that noted in other forms of choroiditis. Our fluorescein findings are exactly the same as those reported by Gass (1968), Amalric and Bonnin (1969), and Van Buskirk and others (1971). We do not tend to agree, however, with the interpretation maintained by Van Buskirk of the absence of choroidal fluorescence in the initial filling stages. We do not think that the initially non-fluorescent patches are due to defective choroidal filling. Like Gass (I968) we believe that the overlying oedematous patches obscure the choroidal fluorescence in the initial stages.

We think that the cellular infiltrates, which are in all probability located in the choriocapillaris and pigment epithelium, tend to obscure the underlying choroidal fluorescence initially and to stain with fluorescein later. The pattern in the scar stage is that of extensive atrophy of the pigment epithelium and pigment migration. In some places there is evidence of atrophy of the choriocapillaris (Fig. 8). In these scar stages the appearance of the fundus may resemble a hereditary dystrophic process because of its bilateral, rather symmetrical, and dry aspect. Because the pigment epithelium appears to be the tissue most significantly affected in this stage, Gass (1968) adopted the name APMPPE. However, we have to bear in mind that this feature is evident only in the scar stage. The fact that some cells may be found in the vitreous and even in the anterior chamber points to an inflammatory reaction. It is possible that this entity reflects an acute inflammation of the choriocapillaris which quickly affects the pigment epithelium without affecting the intermediate and large choroidal vessels. The histologically proven vasculitis in one of our patients who had erythema nodosum and in another case with erythema nodosum described by Van Buskirk and others (197I) supports this assumption. For this reason we should like to suggest as an alternative the term "acute multifocal choriocapillaritis".

However, as pointed out by Gass, a primary affection of the pigment epithelium, unlikely as it may seem, cannot be ruled out completely. The initial aspect of the round whitish placoid lesions, not unlike large soft photocoagulates, may suggest primary pigment epithelium involvement. The severely affected EOG in the acute stage points to a strongly disturbed function of the retinal pigment epithelium. However, this may well be a secondary effect due to widespread choriocapillaritis. In the scar stage the EOG appeared to have markedly recovered. The ERG findings in Case 3, low-normal values in one eye and subnormal values in the other, indicate a variable amount of dysfunction of the photoreceptors (giving rise to the ERG a-waves) and the more superficial retinal layers (giving rise to the ERG b-waves). A severe diffuse involvement of longer standing was reflected by the ERG findings in one eye, 2 months after the onset.

We do not think that this condition is extremely rare, and we think that in most cases a diagnosis of diffuse choroiditis is made. The detailed description provided by Gass ( 1968 ) of this specific reaction pattern enables it to be distinguished from other inflammatory and degenerative choroido-retinal affections. Once the diagnosis is made, the patient can be informed, even in the acute stage of near blindness, of the relatively benign prognosis. 
The cause of this affection remains unknown. No evidence of active tuberculosis, syphilis, histoplasmosis, sarcoidosis, or toxoplasmosis was found. All three patients appeared to be generally healthy. It is worth noticing, however, that two of Gass's cases had a positive tuberculin skin test while the third had a positive family history of tuberculosis; that in the case of Van Buskirk and others (197I) there was erythema nodosum and a positive family history of tuberculosis; and that in one of our cases there was erythema nodosum and in another a positive family history of tuberculosis. We cannot deny a possible link between tuberculosis and the present condition.

Erythema nodosum is supposed to be an allergic vascular reaction due to toxins and is thought to be associated mostly with streptococcal infection, tuberculosis, and sarcoidosis (Borrie, 1967; Weinstein, 1969). So far, erythema nodosum has been found in two cases. According to Borrie (1967), half of all the cases of erythema nodosum are due to streptococcal infection. So far only one patient, in whom there was no erythema nodosum, showed evidence of streptococcal infection (our Case I).

Oral contraceptives were used by only three out of the seven patients who have been described in detail (Gass, I968; Van Buskirk and others, 1971; present study), and do not therefore seem to have any bearing on the matter.

All of the patients reported so far have been young females, but the series is a relatively small one, and this female preponderance may be fortuitous. It is to be noted, however, that erythema nodosum is approximately three times as common in women as in men (Borrie, 1967).

The finding of erythema nodosum in two out of seven extensively examined cases suggests that the present condition may be due to a more or less general vasculitis based on hypersensitivity. Oral cortisone therapy seems therefore to be justified.

\section{Summary}

Three cases of acute posterior multifocal placoid pigment epitheliopathy are presented. Fluorescein angiographic and electrophysiological studies of the retina were performed. No cause of this affection could be demonstrated.

Data are presented which suggest that this entity may be a choriocapillaritis rather than a pigment epitheliopathy.

We should like to thank Dr. H. E. Henkes for allowing us to study one of his cases, Dr. K. Zahn for his help in taking the fluorescence photographs, Dr. G. H. M. van Lith for the electrophysiological tests, and Miss Agnes Wallaart for preparing the manuscript.

\section{References}

AmalRic, P., and BonNin, P. (1969) 'Angiographie fluorescéinique', Bull. Soc. ophtal. Fr., numéro spécial

BORRIE, P. (1967) “Roxburgh's Common Skin Diseases", I $3^{\text {th }}$ ed. Lewis, London

Deutman, A. F. (197I) “The Hereditary Dystrophies of the Posterior Pole of the Eye". Van

Gorcum, Assen

DUKE-ELDER, S., and PERKINS, E. (1966) "System of Ophthalmology, vol. 9: Diseases of the Uveal

Tract". Kimpton, London

GAsS, J. D. M. (1968) Arch. Ophthal. (Chicago), 80, I 77

MaUmenee, A. E. (1970) Amer. F. Ophthal., 69, I

VAN BUSKIRK, E. M., LesSell, s., and FRIEDMAN, E. (1971) Arch. Ophthal. (Chicago), 85, 369

WEINSTEIN, L. (1969) "Disease-a-Month", June, pp. I-30 